that telephone consultation should be on demand and by telephone, 25 (78.1\%) considered it necessary to include video calls and the possibility of receiving reports in non-face to face care and photos. Finally, $23(71.9 \%)$ centers recorded nursing and medical telephone consultations in their medical records.

Conclusion: The pandemic had a huge impact on nursing care for rheumatic patients, with difficulties associated with extra work load and changes in the dynamics of care. A readjustment of assistance has been necessary and audiovisual aids were necessary to improve telephone (non face to face) service.

Disclosure of Interests: None declared

DOI: 10.1136/annrheumdis-2021-eular.1923

\section{AB0905-HPR TELEMEDICINE IN RHEUMATOLOGY AT TIME OF COVID PANDEMIC}

G. Cuomo ${ }^{1}$, F. Masini ${ }^{2}$, K. Gjeloshi², D. Lubrano ${ }^{2}$, L. E. Adinolfi' ${ }^{2}$ C. Romano ${ }^{2}$. ${ }^{1}$ University of Campania Studies "Luigi Vanvitelli", Precision of Medicine, Napoli, Italy; ${ }^{2}$ University of Campania Studies "Luigi Vanvitelli”, Department of Medical and Surgical Sciences, Napoli, Italy

Background: The outbreak of the severe acute respiratory syndrome coronavirus 2 (SARS-CoV-2) pandemic has imposed considerable restrictions on people's mobility in order to limit infection transmission. Before the COVID -19 pandemic tele-rheumatology was proposed to patients living in remote areas. Currently, the use of telemedicine has increased significantly. We report on the implementation of a telemedicine program for the evaluation and treatment of patients with rheumatic diseases (1).

Objectives: The aim of the study is to evaluate telemedicine as a viable approach for routine follow-up and management of rheumatic disease.

Methods: Sixty-six patients were evaluated online by the remote rheumatologist in four weeks. The population of rheumatology patients was evaluated using a IARPLUS platform Information collected included demographic information consisting of age, gender, and primary rheumatologic diagnosis. Results: The average age of patients who were seen was 54 years and $85 \%$ of patients were women.

The most common disorders included rheumatoid arthritis (22, 33\%), axial spondyloarthropathies $(7,11 \%)$, and psoriatic arthritis (15,23\%), systemic sclerosis $(13,20 \%)$, lupus $(6,9 \%)$, UCTD $(2,3 \%)$, Sjogren and fibromyalgia $(1,1.5 \%)$. Results: All patients were given recommendations on COVID-19 vaccine administration. Therapy was remodeled in 13 patients (in particular in 5 patients with anemia intravenous iron infusion was scheduled; 1 started immunosuppressant therapy for proteinuria, 4 increased methotrexate for disease activity, 2 discontinued Methotrexate for adverse events (hypertransaminasemia), 1 suspended $\mathrm{OH}$-chloroquine due to retinal accumulation; 2 biologic-naive patients, after a three-month of waiting due to inability to come to the hospital, started the biologic drug; 22 patients received a renewal of the therapeutic plan; 25 patients had a regular six-month follow up; 2 ticket exemption for illness; 13 consultations and/ or laboratory tests (1 pneumological consultation, 1 ophthalmological consultation, 1 request for sacro-iliac MRI, 3 nailfold videocapillaroscopies, 3 FKT, 1 musculotendinous ultrasound, 1 antibodies for celiac disease, 2 antibodies anti Sars-CoV-2)

Conclusion: Telemedicine is becoming more prevalent. We report the successful use of this service in evaluation and management of rheumatic diseases in a period with limited access to rheumatologic care. We have shown that patients can be seen, evaluated, and successfully treated with a variety of medications, including biologic agents, and evaluated for both chronic inflammatory arthropaties and connective tissue diseases.

\section{REFERENCES}

[1] Rheumatology Care Using Telemedicine Michael M. Rezaian; Telemedicine and e-Health, 2020.

Disclosure of Interests: None declared

DOI: 10.1136/annrheumdis-2021-eular.2719

\section{AB0906-HPR BENEFIT OF A NURSE-LED PROGRAM OF CARE FOR MANAGEMENT OF PATIENTS WITH ESTABLISHED RHEUMATOID ARTHRITIS: SYSTEMATIC LITERATURE REVIEW}

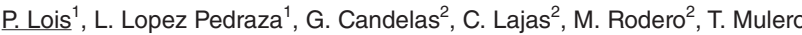
López $^{2}$, L. León ${ }^{1}$, B. Fernandez ${ }^{1}$, L. Rodriguez Rodriguez ${ }^{1}$, L. Abasolo ${ }^{1}$ on behalf of Departamento de Investigación de la Unidad de Gestión Clínica de Reumatología del Hospital Clínico San Carlos. ${ }^{1}$ Hospital Clínico San Carlos, Instituto de Investigación Sanitaria del Hospital Clínico San Carlos (IdISSC), Reumatología, Madrid, Spain; ${ }^{2}$ Hospital Clínico San Carlos, Reumatología, Madrid, Spain

Background: Nowadays, many countries experience an urgent demand for rheumatologists and greater cost-effectiveness. Thus, different alternatives to conventional outpatient physician-led follow-up have been investigated. The alternative of community-based service delivery systems is intended to meet emerging needs, reducing the costs of hospital care and morbidity. In patients with long-term conditions, such as Rheumatoid Arthritis (RA), nurses are seen as essential in the delivery of promotion in self-care and decision-making, favoring the empowerment of patients.

Objectives: To systematically investigate if the nurse led-care management (NLCM) education programs are beneficial in patients with RA.

Methods: This is a systematic literature research publication on the following bibliographic databases: Medline, Embase and the Cochrane Central register of Controlled Trials from the beginning until 2019.

Results: From a yield of 116 studies, 44 were included in the review. After quality assessment and exclusion of the lowest-ranked studies, 19 studies were analyzed. Outcomes were organized into six themes (1) disease activity; (2) adherence; (3)satisfaction; (4)treatment compliance, (5) depression and (6)quality of life. All studies had significant results in at least one of the outcomes.

Conclusion: According to our results, we can conclude that, clinicians could benefit from support conducted by nurse in the management of these patients in tasks such as metrology clinic, participation in enforcement, self-administration, the correct dosage and side effects of treatments, and link the patient and other professionals or entities. It would therefore be advisable to conduct further studies to draw more accurate and reliable conclusions in this context.

\section{REFERENCES:}

[1] Bech B, Primdahl J, Van Tubergen A, et al. 2018 update of the EULAR recommendations for the role of the nurse in the management of chronic inflammatory arthritis. Ann Rheum Dis 2020; 79: 61-68.

[2] Uthman I, Almoallim H, Buckley CD, et al. Nurse-led care for the management of rheumatoid arthritis: a review of the global literature and proposed strategies for implementation in Africa and the Middle East. Rheumatology International 2020; 1: 3

[3] Garner S, Lopatina E, Rankin JA, et al. Nurse-led care for patients with rheumatoid arthritis: A systematic review of the effect on quality of care. $J$ Rheu matol 2017; 44: 757-765.

Disclosure of Interests: None declared

DOI: 10.1136/annrheumdis-2021-eular.3580

\section{AB0907-HPR UNEXPECTED DIFFERENCE IN ACCEPTANCE OF TELECONSULTATION BETWEEN PATIENTS WITH LUPUS AND RHEUMATOID ARTHRITIS WHO UNDERWENT TO A DEVELOPED AND IMPLEMENTED TELEMEDICINE INNOVATIVE PROGRAM AFTER THE DECLARATION OF QUARANTINE DUE TO THE COVID- 19 PANDEMIC IN COLOMBIA}

S. M. Hernández-Zambrano ${ }^{1}$, R. A. Castiblanco-Montañez ${ }^{1}$, J. Chavez-Chavez ${ }^{2}$ D. P. Rivera-Triana ${ }^{2}$, A. Aza $^{3}$, L. Villarreal ${ }^{3}$, M. Martinez ${ }^{4}$, A. Rojas-Villarraga ${ }^{2}$, P. Santos-Moreno ${ }^{5} .{ }^{1}$ Fundacion Universitaria de Ciencias de la Salud - FUCS, Nurse Department, Bogota, Colombia; ${ }^{2}$ Fundacion Universitaria de Ciencias de la Salud - FUCS, Research Division, Bogota, Colombia; ${ }^{3}$ Biomab - Center for Rheumatoid Arthritis, Bogota, Colombia, Administration, Bogota, Colombia; ${ }^{4}$ Biomab - Center for Rheumatoid Arthritis, Bogota, Colombia, General Medicine, Bogota, Colombia; ${ }^{5}$ Biomab - Center for Rheumatoid Arthritis, Bogota, Colombia, Rheumatology, Bogota, Colombia

Background: The Covid-19 pandemic has generated restrictions in the mobility of people, affecting the face-to-face care of patients with chronic diseases, including autoimmune. The health emergency has created the need to establish follow-up alternatives, giving rise to telemedicine.

Objectives: To evaluate the level of attendance to teleconsultation and the faceto-face usual care in patients with rheumatoid arthritis (RA) and systemic lupus erythematosus (SLE) who underwent to a developed innovative telemedicine program after the declaration of quarantine due to the Covid-19 pandemic.

Methods: The present cross-sectional descriptive study reports retrospective data collected from patients with RA and SLE from March to June, 2020;. Since the beginning of March 2020 when Covid-19 was declared in Colombia as a health emergency establishing specific standards for outpatient care, our cente has made the necessary legal and technical adjustments to develop an innovative telemedicine service to prevent the epidemiological risk. To all contacted patients were offered the option of teleconsultation or face-to-face consultation; a standardized protocol was set with clinimetry measures evaluated in both groups. Continuous variables were described using mean and standard deviation, and categorical variables were described using numbers and percentages. We performed chi-square tests of independence to determine differences between teleconsulting and conventional face-to-face consultation.

Results: A total of 5745 RA patients were followed-up, $5292(92.1 \%)$ by teleconsulting and $453(7.9 \%)$ by conventional face-to-face consultation; among the group of SLE patients, a total of 646 were assessed, of which there were 386 $(60 \%)$ by teleconsultation and $260(40 \%)$ by face-to-face consultation; this highlights an important difference in the level of acceptance of the teleconsultation 
between patients with RA and SLE, being much lower in patients with SLE ( $p$ value $<0.0001)$. Regarding gender differences, in men, there were $33(12.7 \%)$ SLE and $89(19.6 \%)$ RA face-to-face consultations, while in women there were $227(87.3 \%)$ SLE and 364 (80.4\%) RA in a face-to-face consultation. Regarding patients who were attended through telemedicine, 45 (11.7\%) SLE and 966 (18.3\%) RA were men, while 341 (88.3\%) SLE and 4326 (81.7\%) RA were women; that means, in the RA cohort, a greater number of men prefer the faceto-face consultation than in the SLE cohort ( $p$ value< 0.0185$)$. 10 RA patients were diagnosed with the Covid-19 in teleconsultation; in all cases, close contact with infected relatives was verified as the probable cause.

Table 1. Differences by gender in the level of teleconsultation acceptance and face-to-face assistance in patients with SLE and RA

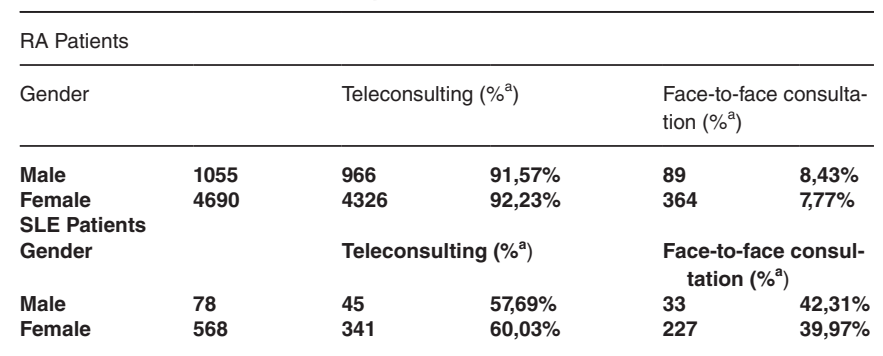

a Total percentage of patients by gender. RA: Rheumatoid arthritis; SLE: Systemic lupus erythematosus.

Conclusion: Telemedicine may provide a viable option for the follow-up of patients with rheumatological diseases even beyond the pandemic. But unexpectedly, data showed an important difference in the acceptance of teleconsultation between patients with RA and SLE, being much higher in older and/or male patients with RA compared to patients with SLE; however, further studies are needed to support this conclusion.

Disclosure of Interests: None declared

DOI: 10.1136/annrheumdis-2021-eular.3899

\section{AB0908-HPR SIGNED, SEALED, DELIVERED: PHARMACISTS ACHIEVING BETTER CARE CLOSER TO HOME IN RHEUMATOLOGY HIGH COST DRUG MANAGEMENT DURING THE COVID-19 PANDEMIC}

S. Gohil ${ }^{1} .{ }^{1}$ University Hospitals of Coventry and Warwickshire NHS Trust, Pharmacy, Coventry, United Kingdom

Background: The Pharmacy High Cost Drug (HCD) and Homecare (HC) team, based at a large acute teaching hospital consists of a range of skilled pharmacists, technicians and administrative staff. The team act as a HCD liaison between the hospital and commissioners, working closely with an expanding variety of specialist clinical teams to manage prescription processing for the following patient groups; HC: 2238; Hospital FP10: 1060; Outsourced Subsidiary Pharmacy Model: 41. HCD management, particularly immunosuppressive biologic therapies for complex rheumatology conditions such as rheumatoid arthritis, has been challenging during the Covid-19 pandemic. The inelasticity in demand for better care closer to home and a functional Integrated Care Records system has intensified, as many patients are now shielding/self-isolating at home. ${ }^{1}$ Frugal innovations have been key during this time - achieving value and simplicity in service re-design to deliver better patient care.

Objectives: This review explores the support provided by the Pharmacy HCD and $\mathrm{HC}$ team to the rheumatology service since March 2020 to aid in service improvements during the pandemic.

Methods: The Pharmacy HCD and HC team reviewed existing practice to better respond to the service changes required during the pandemic in regard to the following areas; identifying patients for Covid-19 shielding; responding to changes in $\mathrm{HC}$ service provision; communication with commissioners; remote staff working; reviewing HCD formulations and administration routes; changes in commissioning arrangements.

Results: i) Rapid development of patient datasets following identification of 1,623 immunosuppressive drug entries in line with BSR Covid-19 guidance (first published in March 2020), thus enabling social distancing/shielding guidance to be issued to patients via letters/virtual platforms. ${ }^{2}$ ii) Swift re-routing of urgent new biologic $\mathrm{HC}$ registrants to a new hospital pharmacy home delivery provision when certain $\mathrm{HC}$ providers temporarily imposed restrictions on intake of new registrants. This hospital initiative was further enhanced to a hub and spoke model enabling patients to have easy access to their medications from their nominated local community pharmacy branch. iii) Providing assurance to commissioners that clinical follow up reviews could continue virtually, blood samples could be taken at an external hub and blueteq documentation would be completed covering NICE technology appraisal (TA) criteria. iv) A successful pilot utilising the lean PDSA (Plan, Do, Study, Act) quality improvement methodology, to enable remote working pharmacists and technicians to process $\mathrm{HC}$ prescriptions remotely in the absence of an electronic prescribing and medicines administration system. v) Reviewing potential plans to switch certain hospital day-case intravenous biosimilar infusions such as infliximab to subcutaneous administration via HC. vi) Business contingency development plans to protect fragile medication supply chains. vii) Clarifying implications for changes in contracting and payment arrangements announced by NHS England and NHS Improvement due to the pandemic. ${ }^{3}$

Conclusion: In summary, the role of the HCD Pharmacists during the pandemic in supporting rheumatology patients and the multidisciplinary team has been fundamental; ensuring signed prescriptions are securely processed to safely deliver medication to those in need.

\section{REFERENCES:}

[1] NHS. The NHS long term plan. 2019. [Online].

[2] British Society for Rheumatology (2020) Covid-19 - Identifying patients for shielding in England. [Online].

[3] NHS. Revised arrangements for NHS contracting and payment during the COVID-19 pandemic. 2020. [Online].

Acknowledgements: Mark Easter, UHCW and Interim Integrated Care System Chief Pharmacist, Hardeep Bagga, Deputy Chief Pharmacist, UHCW Pharmacy Homecare Team and UHCW Specialist Rheumatology Clinical Team.

Disclosure of Interests: None declared

DOI: 10.1136/annrheumdis-2021-eular.3944

\section{AB0909-HPR COVID 19 CRISIS: RAPID DEVELOPMENT OF REMOTE RHEUMATOLOGY MEDICATION CLINICS}

R. Penford ${ }^{1}$, E. Wren ${ }^{1}$, K. Mackay ${ }^{1} .{ }^{1}$ Torbay Hospital, Rheumatology, Torquay, United Kingdom

Background: We used to initiate $\operatorname{DMARD}(\mathrm{s})$ and Biologic therapies via nurseled shared medical appointments (Group clinics) and could see up to 30 patients per week, with a maximum of 6 patients per group. We did this to start patients on their medications efficiently and safely and to manage the increase in workload.

However, with the onset of the COVID 19 pandemic, we had to stop these clinics immediately, but, we did not have capacity to start everyone on their medications in a timely manner by telephone. Telephoning each individual took $>9$ hours per week, whereas previously it took 3-4 haours.

Objectives: We wanted to start patients on their all rheumatology medications safely and efficiently (within 10 days).

Methods: By April 2202, we had organised the filming of 10 short healthcare videos to give patients all the information they required to start a range of DMARDs and biologics.

We developed a new protocol (fig 1); patients are asked to view the relevan video, contact our department to confirm they understand the safety monitoring, risks, potential side effects, dose increases etc. As soon as they confirm by email they are happy to start treatment, a prescription is generated and emailed to the hospital outpatient pharmacy, where it is dispensed and delivered to the patient's home. We send a follow up reminder letter about blood test monitoring etc (copy to GP) and a 'shared care agreement' to GP. They are given the option to have a telephone clinic appointment with a specialist nurse if required.

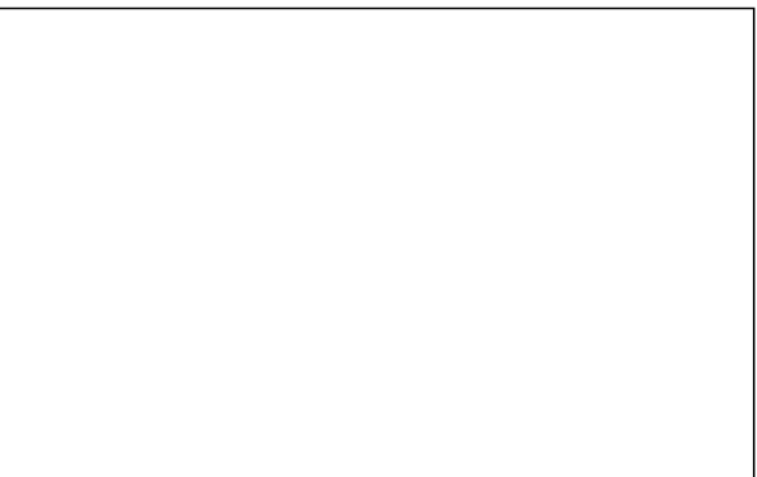

Figure 1.

Results: Of those requiring DMARDs, $62 \%$ reviewed the video, completed the checklist and confirmed by email they were happy to start treatment, within 24-hours. $88 \%$ had completed within 7 days. 